\title{
Measurement of exhaled volatile organic compounds from patients with chronic obstructive pulmonary disease (COPD) using closed gas loop GC-IMS and GC-APCI-MS
}

\author{
M. Allers ${ }^{1}$, J. Langejuergen ${ }^{1}$, A. Gaida ${ }^{2,3}$, O. Holz ${ }^{4}$, S. Schuchardt ${ }^{5}$, J.M. Hohlfeld ${ }^{4}$, S. Zimmermann ${ }^{1}$ \\ ${ }^{1}$ Leibniz Universität Hannover, Institute of Electrical Engineering and Measurement Technology - \\ Hannover/D, \\ ${ }^{2}$ Fraunhofer Institute for Toxicology and Experimental Medicine ITEM, Clinical Airway Research - \\ Hannover/D, \\ ${ }^{3}$ Leibniz Universität Hannover, Institute of Inorganic Chemistry, Research Group Analytical \\ Chemistry - Hannover/D, \\ ${ }^{4}$ Fraunhofer Institute for Toxicology and Experimental Medicine ITEM, Clinical Airway Research, \\ Member of the German Center for Lung Research (BREATH) - Hannover/D, \\ ${ }^{5}$ Fraunhofer Institute for Toxicology and Experimental Medicine ITEM, Bio- and Environmental \\ Analytics - Hannover/D
}

Email corresponding author: $\underline{\text { allers@geml.uni-hannover.de }}$ 


\begin{abstract}
Due to its high sensitivity, compact size and low cost Ion Mobility Spectrometry (IMS) has the potential to become a point-of-care breath analyzer. Therefore, we developed a prototype of a compact, closed gas loop IMS with gas chromatographic (GC) pre-separation and high resolving power of $\mathrm{R}=90$. In this study, we evaluated the performance of this GC-IMS under clinical conditions in a COPD study to find correlations between VOCs $\left(10 \mathrm{ppb}_{\mathrm{v}}\right.$ to $\left.1 \mathrm{ppm}_{\mathrm{v}}\right)$ and COPD. Furthermore, in order to investigate possible correlations between ultra-low concentrated breath VOCs $\left(0.1 \mathrm{ppt}_{\mathrm{v}}\right.$ to $1 \mathrm{ppb}_{\mathrm{v}}$ ) and COPD, a modified mass spectrometer (MS) with atmospheric pressure chemical ionization (APCI) and GC pre-separation (GC-APCI-MS) was used. The GC-IMS has been used in 58 subjects (21 smokers with moderate COPD, 12 ex-smokers with COPD, 16 healthy smokers and 9 nonsmokers). GC-APCI-MS data were available for 94 subjects ( 21 smokers with moderate COPD, 25 exsmokers with COPD, 25 healthy smokers and 23 non-smokers). For 44 subjects, a comparison between GC-IMS and GC-APCI-MS data could be performed. Due to service intervals, subject availability and corrupt data, patient numbers were different for GC-APCI-MS and GC-IMS measurements. Using GC-IMS, three VOCs have been found showing a significant difference between healthy controls and patients with COPD. In the GC-APCI-MS data, we only observed one distinctive VOC, which has been identified as 2-pentanone. This proof-of-principle study shows the potential of our high-resolution GC-IMS in the clinical environment. Due to different linear dynamic response ranges, the data of GC-IMS and GC-APCI-MS were only comparable to a limited extent.
\end{abstract}

Keywords: COPD, GC-IMS, GC-APCI-MS, VOC, biomarker, breath analysis 


\section{Introduction}

The analysis of volatile organic compounds (VOCs) in exhaled breath has great potential to noninvasively diagnose and monitor respiratory and systemic diseases. Many different technologies are available and currently used to analyze breath VOCs, such as sensor based electronic noses [1, 2], gas chromatography - electron ionization - mass spectrometry (GC-EI-MS) [3, 4], selected ion flow tubemass spectrometry (SIFT-MS) [5], proton transfer reaction mass spectrometry (PTR-MS) [6, 7] or ion mobility spectrometry (IMS) with gas chromatographic pre-separation by a multi-capillary column (MCC) $[8,9]$.

At present, the results of the different methods are hard to compare, but most of them have been able to show differences between COPD patients and controls or other diseases like asthma or cancer [1015]. For example, it is already shown [14], that the BioScout MCC-IMS (B\&S Analytik, Dortmund, Germany) provides sufficient sensitivity to detect specific COPD related VOCs. However, in order to reach this sensitivity, a high sample volume in the range of $10-50 \mathrm{~mL}$ is necessary, which can be achieved by MCC with comparatively high flow rate and high sample capacity [16]. Nonetheless, the MCC separation power is limited, which might lead to chemical cross sensitivities in the IMS due to co-eluting compounds. Moreover, because of the low mobility resolving power of the IMS [17], two or more analytes with similar drift times might not be separated. Furthermore, the systems need continuous flow of dry and clean drift gas provided by a gas cylinder or laboratory gas supply.

Since IMS has the potential to be further developed towards point-of-care applications, we developed a prototype of a compact, closed gas-loop GC-IMS [18]. Compared to the IMS used in the BioScout MCC-IMS, which has a mobility resolving power of about $\mathrm{R}=15$ (Drift time/Full Width at Half Maximum (FWHM)) [17], our IMS combines much higher mobility resolving power of $\mathrm{R}=90$ with highest sensitivity [19]. Due to its high sensitivity, only $0.2 \mathrm{~mL}$ of breath are required. Thus, the IMS can be coupled to a standard GC capillary column for pre-separation providing better separation power as compared to MCC. Hence, cross sensitivity is decreased. In addition, the system is equipped with a filter recirculation system and does not need any external gas supply. Since this prototype has not been used in a clinical study yet, our objective is to evaluate this system in a clinical COPD study.

Another focus of our work is to investigate possible correlations between ultra-low concentrated breath VOCs $\left(0.1 \mathrm{ppt}_{\mathrm{v}}\right.$ to $\left.1 \mathrm{ppb}_{\mathrm{v}}\right)$ and COPD by using a modified ultra-high sensitive mass spectrometer with atmospheric pressure chemical ionization and GC pre-separation (GC-APCI-MS) $[20,21]$. It is reported [22] that for trace components, the ionization efficiency of APCI is $10^{3}-10^{4}$ times greater than that of electron "impact" ionization (EI) at reduced pressures used in standard GCMS systems. Thus, another aim of this work is to gather additional information from ultra-low concentrated breath VOCs not detectable with standard GC- MS using practical breath sample volumes or GC-IMS. Furthermore, the GC-APCI-MS data are compared with GC-IMS data. Since both systems use the same atmospheric pressure chemical ionization, we expect some correlations between both data sets.

The two different analysis technologies have been used in a large COPD study that was performed in two German centers for lung research ("Biomedical Research in Endstage and Obstructive Lung Disease Hannover (BREATH)" and "Universities Giessen and Marburg Lung Centre (UGMLC)"). The COPD study is described in detail by Gaida et al. [23]. 


\section{Methods}

\subsection{Experimental setup}

\subsubsection{GC-IMS}

For the breath measurements presented in this work, a novel, compact, closed gas loop high-resolution IMS was used. A detailed description of the system can be found elsewhere [18]. The two main components are a drift tube IMS and an isothermal GC oven with a $10 \mathrm{~m}$ standard capillary column (Restek, RTX volatiles, ID of $530 \mu \mathrm{m}$, film thickness of $2 \mu \mathrm{m}$ ). The GC-IMS is equipped with a filter recirculation system providing both a continuous supply of dry and clean drift gas and GC carrier gas, so that no external gas supply is needed. All relevant operating parameters are summarized in online supplement 1 .

The IMS with a length of $7.5 \mathrm{~cm}$ achieves a mobility resolving power of $\mathrm{R}=90$ (Drift time/FWHM) at a drift voltage of $5 \mathrm{kV}$ [19]. A radioactive electron source $\left(300 \mathrm{MBq}{ }^{3} \mathrm{H}\right)$ is used for ionization. It operates in the positive mode at $40{ }^{\circ} \mathrm{C}$ and 10 mbar above ambient pressure. The drift gas flow is $150 \mathrm{sccm}$. During operation a defined sample gas flow of $100 \mathrm{sccm}$ is pumped from the sample inlet through a sample loop with a volume of $0.2 \mathrm{~mL}$. The sample loop is injected via a 6-port-valve into the GC carrier gas stream. The carrier gas flow is $4 \mathrm{sccm}$ for the first $20 \mathrm{~min}$. After $20 \mathrm{~min}$ the flow rate is increased to $20 \mathrm{sccm}$ (conditioning flow rate) for $25 \mathrm{~min}$ (conditioning time) in order to elute all remaining compounds. The $\mathrm{GC}$ is operated at a constant temperature of $\mathrm{T}=50^{\circ} \mathrm{C}$. After passing the GC, the carrier gas flows into the IMS ionization region. Thus, all peaks found in GC-IMS measurements are characterized by their IMS drift time, GC retention time and peak area, which relates to the compound concentration.

In order to determine the detection limit and linear dynamic response range of the GC-IMS, acetone has been used as a model substance for dry conditions. Of course, the results will differ for other compounds depending on their proton and electron affinities, and retention times. For the acetone dimer, a detection limit of $6.9 \mathrm{ppb}_{\mathrm{v}}$ has been calculated. As seen in Figure 1, the linear dynamic response range with respect to the acetone dimer spans from $10 \mathrm{ppb}_{\mathrm{v}}$ to $1 \mathrm{ppm}_{\mathrm{v}}$. Since ionization is based on APCI, the acetone intensity is strongly moisture-dependent. However, in [24] it is shown, that the chosen GC parameter allow an adequate separation of water and acetone. Thus, the moisture effect can be neglected in our GC-IMS for acetone and for all compounds with even longer retention times. 


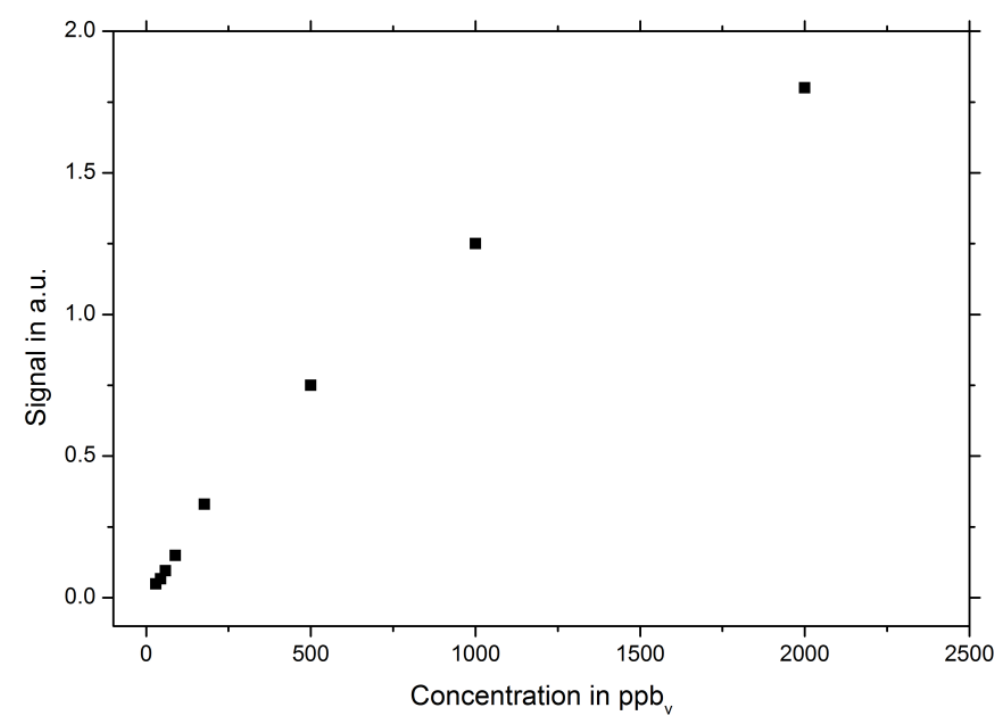

Figure 1: Calibration curve of acetone measured by GC-IMS.

\subsubsection{GC-APCI-MS}

In addition to the GC-IMS, a GC-APCI-MS was used [20, 21]. Loaded Tenax TA adsorption tubes were therefore heated up to $180{ }^{\circ} \mathrm{C}$ for $5 \mathrm{~min}$ in a thermal desorption system (Gerstel TDS 3). A constant flow of $23 \mathrm{sccm}$ nitrogen was used to flush the tubes. $4 \mathrm{sccm}$ of it were directed through a cryo trap which was cooled by liquid nitrogen to $-10^{\circ} \mathrm{C}$. A Tenax TA liner was used for trapping. After $8.5 \mathrm{~min}$ the cold injection system (Gerstel CIS 4) was heated up to $200{ }^{\circ} \mathrm{C}$ with a rate of $12 \mathrm{~K} / \mathrm{s}$ and the desorbed gas was injected into the GC (Agilent 7890) at a flow rate of $3 \mathrm{sccm}$. The initial GC temperature was $30^{\circ} \mathrm{C}$. It was increased to $220^{\circ} \mathrm{C}$ after $10 \mathrm{~min}$. of acquisition. The $\mathrm{GC}$ column was a 30 meter RTX-Volatiles (Restek) with an inner diameter of $530 \mu \mathrm{m}$ and a film thickness of $2 \mu \mathrm{m}$. The GC gas flow was further diluted with approx. $600 \mathrm{sccm}$ of dry air and then ionized in our atmospheric pressure chemical ionization (APCI) source using a radioactive $300 \mathrm{MBq}{ }^{3} \mathrm{H}$ source identical to the source used in our IMS. The ionized sample gas was then transferred via gas flow and electric fields into a time-of-flight mass spectrometer (Bruker micrOTOF II). All relevant parameters are summarized in online supplement 2 .

Since different detection limits and in particular different linear dynamic response ranges of both systems (GC-IMS and GC-APCI-MS) affect the interpretation of our experimental results, below these parameters are also provided for the GC-APCI-MS. However, for better understanding of our experimental results, the different analytical methods should be compared not only by the detection limits of the analyzers, but by including sample pre-concentration, thermal desorption effects and preseparation resolving power.

The detection limit and the linear dynamic response range of the GC-APCI-MS were determined based on acetone as model substance. Again, the results will differ for other compounds depending on their proton and electron affinities, retention times and adsorption characteristic. Due to the soft and more efficient ionization process at atmospheric pressure for most substances and the significant lower noise of the MS detector (Micro-channel plate) in comparison to the IMS detector (Faraday plate), ultra-low detection limits in the $\mathrm{ppq}_{\mathrm{v}}$-range can be obtained with APCI-MS using a direct sample inlet. For example, the detection limit of acetone in clean dry air is about $600 \mathrm{ppq}_{\mathrm{v}}$ for APCI-MS [25].

However, in this study, the APCI-MS is coupled to a thermal desorption system (TDS) and a gas chromatograph (GC) for pre-separation. Thus, pre-concentration using adsorption tubes and resolving 
power of the GC depending on column length, column flow, column temperature etc. influence the detection limits in this setup (GC-APCI-MS). In this case, a detection limit of $400 \mathrm{ppq}_{\mathrm{v}}$ was determined for acetone with respect to the whole measurement process. The resulting calibration curve is given in Figure 2. Due to a significant higher sample volume concentrated on the Tenax TA tubes compared to the sample volume used in the GC-IMS, the linear dynamic response range shifts towards lower concentrations. The linear response for acetone just reaches up to $5 \mathrm{ppb}_{\mathrm{v}}$ for the GC-APCI-MS, which is below the detection limit of the GC-IMS. Thus, for the breath measurements in this study, two systems are available differing in their linear dynamic response ranges and detection limits which can lead to different correlations of VOCs in breath and COPD.

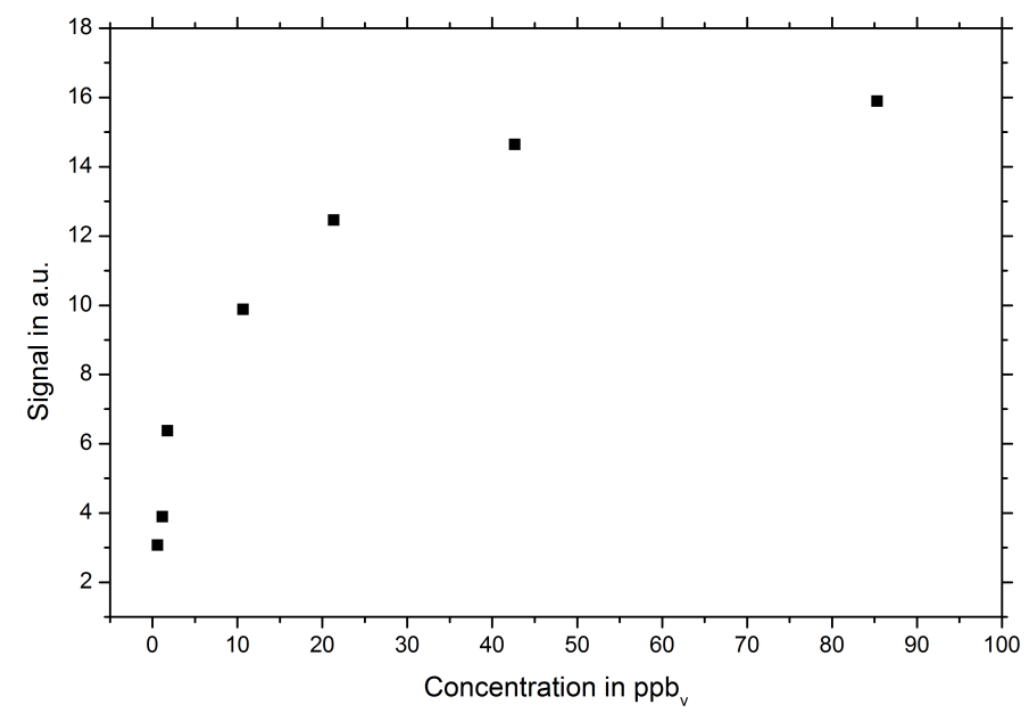

Figure 2: Calibration curve of acetone measured by GC-APCI-MS.

\subsection{Subjects}

The GC-IMS has been used in 58 subjects (21 smokers with moderate COPD, 12 ex-smokers with COPD, 16 healthy smokers and 9 non-smokers). GC-APCI-MS data are available for 94 subjects (21 smokers with moderate COPD, 25 ex-smokers with COPD, 25 healthy smokers and 23 non-smokers). For 44 subjects, a comparison between GC-IMS and GC-APCI-MS data could be performed. Due to service intervals, subject availability and corrupt data, patient numbers are different for GC-APCI-MS and GC-IMS measurements. However, Table 1 shows that the demographics of subjects available for the analysis by GC-IMS and by GC-APCI-MS were comparable. Subjects had to be free of exacerbations or acute infections within four weeks prior to the study day. The study was conducted in accordance with Good Clinical Practice and the Declaration of Helsinki. Subjects gave their written informed consent. The study was approved by the Ethical Committees of Hannover Medical School (AZ 6490). 
Table 1: Subjects demographics. FVC: forced vital capacity, \%pred=\% of predicted normal, FEV1:=forced expiratory volume in 1 second, FeNO:=level of exhaled nitric oxide, $\mathrm{CO}:=$ exhaled carbon dioxide, $\mathrm{COHb}:=$ calculated carboxyhemoglobin from exhaled $\mathrm{CO}$ analysis, Mean \pm SD are given.

\begin{tabular}{|l|c|c|c|c|c|c|c|c|}
\hline \multirow{2}{*}{} & \multicolumn{4}{|c|}{ GC-IMS } & \multicolumn{3}{c|}{ TD-APCI-GC-MS } \\
\cline { 2 - 9 } & Non/Ex-smoker & \multicolumn{2}{|c|}{ Smoker } & \multicolumn{2}{c|}{ Non/Ex-smoker } & \multicolumn{2}{c|}{ Smoker } \\
\cline { 2 - 9 } & healthy & COPD & healthy & COPD & healthy & COPD & healthy & COPD \\
\hline $\mathbf{n}$ & 9 & 12 & 16 & 21 & 23 & 25 & 25 & 21 \\
\hline Gender (f/m) & $5 f / 4 m$ & $9 f / 3 m$ & $7 f / 9 m$ & $12 f / 9 m$ & $14 f / 9 m$ & $12 f / 13 m$ & $15 f / 10 m$ & $9 f / 12 m$ \\
\hline Age (y) & $44 \pm 16$ & $65 \pm 05$ & $49 \pm 11$ & $62 \pm 07$ & $37 \pm 14$ & $65 \pm 05$ & $46 \pm 12$ & $62 \pm 06$ \\
\hline Height (cm) & $173.8 \pm$ & $165.8 \pm$ & $170.9 \pm$ & $171.7 \pm$ & $171.7 \pm$ & $169.9 \pm$ & $169.5 \pm$ & $172.8 \pm$ \\
& 8.3 & 8.2 & 9.8 & 10.8 & 8.0 & 9.8 & 8.7 & 11.6 \\
\hline Weight (kg) & $69.3 \pm$ & $79.6 \pm$ & $76.8 \pm$ & $78.2 \pm$ & $68.7 \pm$ & $85.0 \pm$ & $73.7 \pm$ & $80.7 \pm$ \\
& 10.3 & 14.0 & 18.4 & 12.2 & 13.4 & 16.5 & 17.3 & 17.1 \\
\hline BMI & $22.8 \pm$ & $28.8 \pm$ & $26.1 \pm 4.9$ & $26.5 \pm 3.1$ & $23.2 \pm 3.6$ & $29.4 \pm 5.1$ & $25.5 \pm$ & $26.8 \pm 3.8$ \\
& 2.0 & 3.5 & & & & & 4.7 & \\
\hline FVC(L) & $4.8 \pm 1.0$ & $1.6 \pm 1.7$ & $4.4 \pm 0.8$ & $3.1 \pm 1.9$ & $4.5 \pm 1.0$ & $2.6 \pm 1.8$ & $4.3 \pm 0.7$ & $3.1 \pm 2.1$ \\
\hline FVC(\%pred.) & $4.8 \pm 1.0$ & $1.6 \pm 1.7$ & $4.4 \pm 0.8$ & $3.1 \pm 1.9$ & $110.2 \pm$ & $77.5 \pm$ & $115.1 \pm$ & $86.3 \pm$ \\
& & & & & 13.7 & 54.9 & 16.6 & 52.6 \\
\hline FEV1(L) & $3.7 \pm 0.8$ & $0.9 \pm 1.0$ & $3.3 \pm 0.7$ & $1.8 \pm 1.2$ & $3.6 \pm 0.8$ & $1.3 \pm 1.0$ & $3.2 \pm 0.6$ & $1.7 \pm 1.2$ \\
\hline FEV1(\%pred.) & $109.4 \pm$ & $36.1 \pm$ & $104.5 \pm$ & $64.4 \pm$ & $104.7 \pm$ & $46.9 \pm$ & $103.9 \pm$ & $58.8 \pm$ \\
& 9.0 & 43.8 & 13.1 & 37.4 & 12.0 & 36.9 & 12.4 & 38.9 \\
\hline FEV1/ FVC & $77.5 \pm$ & $26.9 \pm$ & $75.7 \pm 4.3$ & $46.1 \pm$ & $80.7 \pm 4.8$ & $34.4 \pm$ & $75.8 \pm$ & $41.4 \pm$ \\
(\%) & 4.8 & 28.6 & & 24.7 & & 23.8 & 4.8 & 25.3 \\
\hline FeNO (ppb) & 16.0 & 21.8 & $10.8(9.5)$ & $9.5(5.0)$ & $15.0(8.0)$ & 21.5 & $9.0(5.0)$ & $10.5(7.0)$ \\
& $(5.0)$ & $(11.9)$ & & & & $(16.5)$ & 2.0 & 10.0 \\
\hline CO & $2.0(3.0)$ & $2.0(0.5)$ & $10.0(3.0)$ & 10.0 & $2.0(2.0)$ & $2.0(2.0)$ & 10.0 & 10.0 \\
& & & & $(16.0)$ & & & $(5.0)$ & $(13.0)$ \\
\hline COHb & $0.9(1.1)$ & $0.9(0.3)$ & $2.2(0.5)$ & $2.2(2.6)$ & $0.9(0.4)$ & $0.9(0.4)$ & $2.2(0.8)$ & $2.2(2.1)$ \\
\hline
\end{tabular}

\subsection{Study design and collection of breath samples}

The study was from December 2013 to August 2015. Subjects visited the site on a single day. After undergoing thorough physical examination and providing informed consent, subjects were asked to give breath samples. At first, exhaled carbon monoxide (Smokelyzer, Bedfont, Kent, UK) was analyzed to verify the smoking status. Then, for the collection of breath samples for GC-IMS analysis subjects inhaled through an A2 carbon filter and exhaled into a stainless steel tube sampling reservoir (50 cm length, $4 \mathrm{~cm}$ inner diameter) under exhalation flow control ( $3 \mathrm{~min}$ pre-sampling $/ 5 \mathrm{~min}$ sampling time). For the GC-IMS measurements, the exhaled breath was continuously pumped with $100 \mathrm{sccm}$ from the reservoir through the sample loop of the GC-IMS. The content of the sample loop was injected into the GC carrier gas.

Immediately afterwards, subjects were asked to exhale into a setup using a $\mathrm{CO}_{2}$ triggered (4\%) pump to sample alveolar breath. The subjects used the same mouth piece with A2 filter inhalation. After at least three inhalation/exhalation cycles to remove accumulated environmental VOCs from the lung, 50 $\mathrm{mL}$ of breath were continuously drawn onto a Tenax TA tube.

\subsection{Identification of VOCs using reference substances}

A subset of 19 compounds was chosen for external standard validation and identification. The reference compounds chosen for external standard measurements included alcohols, aldehydes, ketones and aromatic compounds. Defined test gas concentrations in the $\mathrm{ppb}_{\mathrm{v}}$ range were generated via permeation tubes and subsequent gas dilution. These test gases were analyzed using both GC-IMS and 
GC-APCI-MS, in order to determine GC-IMS retention times, IMS drift times and GC-APCI-MS retention times, see Table 2. To compare the results with other IMS devices, the reduced mobilities were calculated. However, it has to be noted, that several parameters besides temperature and pressure affect the ion mobility. Thus, reduced ion mobilities of different IMS devices do not necessarily match.

Table 2: List of reference compounds analyzed by GC-IMS and GC-APCI-MS.

\begin{tabular}{|l|c|c|c|c|}
\hline \multicolumn{1}{|c|}{ Compound } & CAS-Number & $\begin{array}{c}\text { GC-IMS } \\
\text { retention time in s }\end{array}$ & $\begin{array}{c}\text { Reduced ion } \\
\text { mobility in } \\
\mathbf{c m}^{2} / \mathbf{V} \mathbf{s}\end{array}$ & $\begin{array}{c}\text { GC-APCI-MS } \\
\text { retention time in s }\end{array}$ \\
\hline Ethanol & $64-17-5$ & 40 & 1.90 & 402 \\
\hline 2-Propanol & $67-63-0$ & 43 & 1.80 & 515 \\
\hline Isoprene & $78-79-5$ & 44 & 1.83 & 525 \\
\hline Acetone & $67-64-1$ & 46 & 1.74 & 541 \\
\hline Acetonitrile & $75-05-8$ & 48 & 1.92 & 581 \\
\hline 2,3-Butanedione & $431-03-8$ & 63 & 1.67 & 881 \\
\hline Butanal & $123-72-8$ & 66 & $1.67+1.51$ & 889 \\
\hline 2-Butanone & $78-93-3$ & 69 & 1.86 & 909 \\
\hline 1-Butanol & $71-36-3$ & 106 & 1.65 & 1103 \\
\hline 2-Pentanone & $107-87-9$ & 116 & 1.74 & 1207 \\
\hline Pentanal & $110-62-3$ & 127 & $1.59+1.35$ & 1241 \\
\hline 1-Pentanol & $71-41-0$ & 211 & 1.54 & 1434 \\
\hline Toluol & $108-88-3$ & 223 & $1.91+1.73$ & 1531 \\
\hline 2-Hexanone & $591-78-6$ & 266 & 1.63 & 1568 \\
\hline Hexanal & $66-25-1$ & 278 & 1.50 & 1795 \\
\hline 1-Hexanol & $111-27-3$ & 478 & 1.45 & 1919 \\
\hline 3-Heptanone & $106-35-4$ & 556 & $1.56+1.21$ & 1948 \\
\hline 2-Heptanone & $110-43-0$ & 570 & $1.52+1.17$ & 2111 \\
\hline Cyclohexanone & $108-94-1$ & 701 & $1.67+1.32$ & \\
\hline
\end{tabular}

\subsection{Data analysis}

\subsubsection{GC-IMS data}

In order to find biomarkers discriminating COPD patients from healthy controls, the GC-IMS samples were investigating by univariate analysis. In the first step, data from GC-IMS were pre-processed, such as each dataset of a breath sample is represented by a matrix of signal intensities where the $\mathrm{x}$-axis indicates the IMS drift time in milliseconds, the y-axis indicates the GC retention time in seconds and the matrix values indicate signal intensities in volts. In Figure 3, an exemplary excerpt from the GCIMS data of a COPD smoker is shown. 


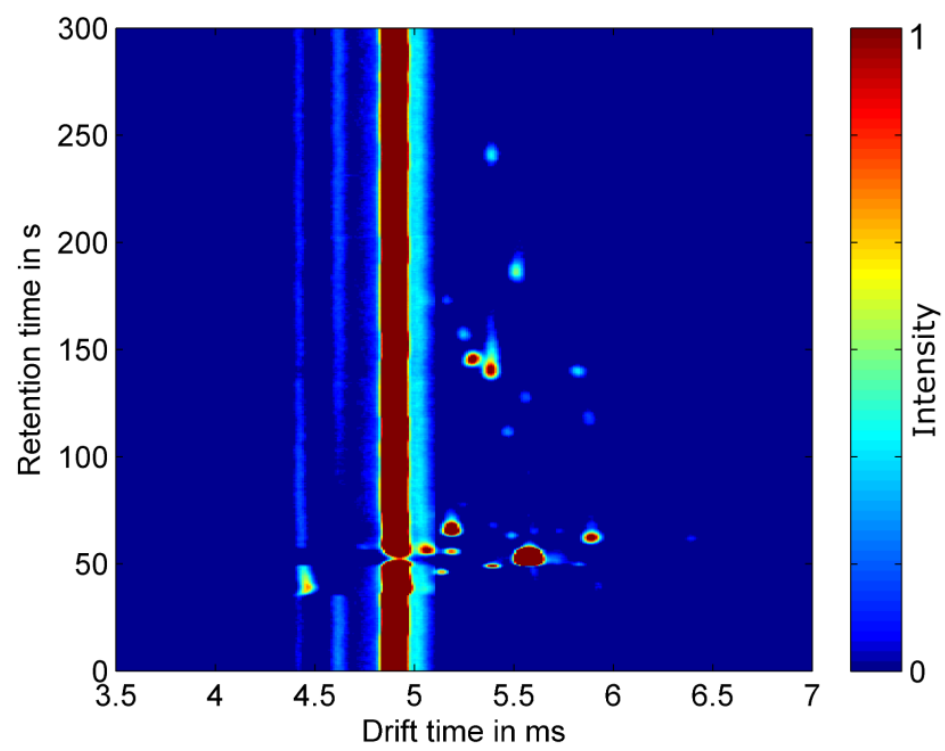

Figure 3: Excerpt from the GC-IMS data of a COPD smoker.

Due to variations in atmospheric pressure, both IMS drift time as well as GC retention time changed slightly from day to day. Since both the IMS and the GC temperature are controlled and the moisture content of the IMS drift gas and GC carrier gas is constant over months, the influence of these parameters on drift and retention time is negligible. Furthermore, the IMS pressure was automatically logged in every measurement for IMS drift time corrections. The retention time was corrected by linear alignment based on peaks that appear in every breath measurement, e.g. Acetone.

In the second step of the univariate analysis, peaks were determined using a peak finder algorithm written in MATLAB. A signal was classified as a peak, when the intensity of the signal was 10 times higher than the standard deviation of the noise. In order to exclude outliers, only peaks appearing in at least one group (COPD smokers, COPD ex-smokers, healthy smokers and healthy non-smokers) in at least 50 percent of all measurements were considered. In the last step, a statistical analysis is conducted based on 45 GC-IMS peaks (classified as explained above) applying Welch's t-test.

\subsubsection{GC-APCI-MS data}

The GC-APCI-MS data were analyzed as described in 2.5.1. The dataset of one breath sample is represented by a matrix of signal intensities where the $\mathrm{x}$-axis indicates the mass-to-charge ratio in $\mathrm{m} / \mathrm{z}$, the $y$-axis indicates the GC retention time in seconds and the matrix values indicate signal intensities in counts. Figure 4 shows an excerpt from the GC-APCI-MS data of the COPD smoker. Due to different GC parameters (e.g. flow rate, temperature, length) the retention time span of $0-25$ min of the GC-APCI-MS fits to the retention time span of 0 - 300 s of the GC-IMS. 


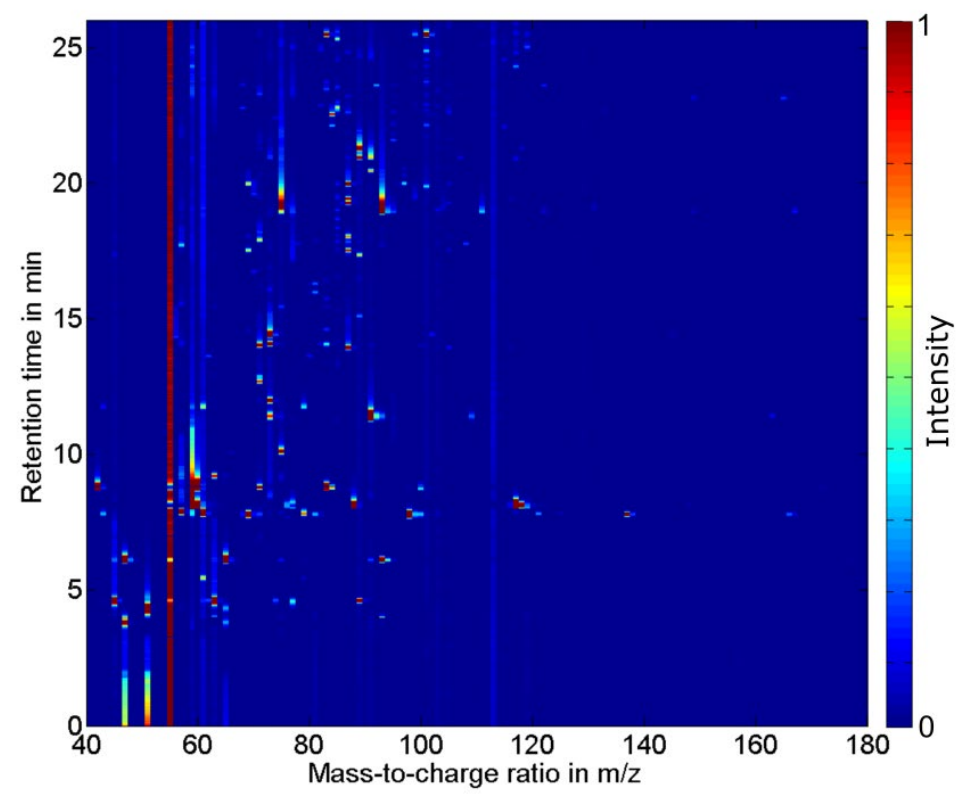

Figure 4: GC-APCI-MS data of a COPD smoker.

Comparing Figure 3 and Figure 4, it can be seen, that the GC-APCI-MS data includes considerably more peaks. This is due to the 250 -fold higher sample volume of $50 \mathrm{~mL}$ that was collected onto the Tenax TA tube. The univariate analysis of the GC-APCI-MS data was performed as described above. In total 102 peaks (classified as explained above) were analyzed using Welch's t-test.

\subsection{Statistical analysis}

Welch's t-test was applied to compare the four groups (COPD smokers, COPD ex-smokers, healthy smokers and healthy non-smokers) with respect to all peaks detected. Welch's t-test is an adaptation of Student's t-test and is more reliable in case of unequal variances and unequal sample sizes. For all tests, a 2 -sided significance level of $1 \%$ was applied, unless otherwise noted. Due to the exploratory nature of this study, no further adjustment for multiple testing was done. The data of breath VOCs are displayed as median, interquartile range (IQR) and whisker with a maximum length of the 1.5 -fold IQR range for every group in the respective figures.

\section{Results}

\section{$3.1 G C-I M S$}

45 GC-IMS VOC peaks were included into the statistical analysis. In Table 3, the 20 most abundant compounds with respect to the mean intensity per subject are shown. Two or more peaks having the same retention time are associated to one compound, since the peaks are assumed to be monomer and dimer peak. Compounds which have not been identified so far are numbered consecutively. 
Table 3: Results of Welch's t-test applied to the GC-IMS data considering the 20 most abundant peaks.

\begin{tabular}{|c|c|c|c|c|}
\hline Compound & $\begin{array}{c}\text { GC } \\
\text { retention time } \\
\text { in } s\end{array}$ & $\begin{array}{c}\text { Reduced ion } \\
\text { mobility in } \\
\mathrm{cm}^{2} / \mathrm{Vs}\end{array}$ & $\begin{array}{c}\text { p-value }<0.01 \\
\text { Smoker vs. } \\
\text { non-smoker }\end{array}$ & $\begin{array}{c}\text { p-value }<0.01 \\
\text { COPD vs. } \\
\text { healthy }\end{array}$ \\
\hline 1 & 35 & 1.64 & & \\
\hline Ethanol & 40 & 1.90 & $\mathrm{X}$ & \\
\hline 2-Propanol & 43 & 1.80 & & \\
\hline 4 & 43.5 & 1.67 & & \\
\hline Acetone & 46 & 1.74 & & \\
\hline 6 & 48 & 1.88 & $\mathrm{X}$ & \\
\hline Acetonitrile & 48.5 & 1.92 & $\mathrm{X}$ & \\
\hline 8 & 49 & 1.80 & & \\
\hline 9 & 54.5 & 1.65 & & \\
\hline 10 & 57 & $1.77+1.69$ & $\mathrm{X}$ & \\
\hline 11 & 58 & 1.88 & & \\
\hline 2-Butanone & 69 & 1.86 & $\mathrm{X}$ & \\
\hline 13 & 101 & 1.78 & & \\
\hline 1-Butanol & 106 & 1.65 & & \\
\hline 15 & 108 & 1.87 & & \\
\hline 2-Pentanone & 116 & 1.74 & & \\
\hline 17 & 127 & $1.80+1.67$ & & \\
\hline 18 & 142 & 1.85 & $\mathrm{X}$ & \\
\hline 19 & 169 & $1.76+1.54$ & & $X$ \\
\hline 20 & 219.5 & $1.80+1.49$ & & $X$ \\
\hline
\end{tabular}

In active smokers, we found significantly $(\mathrm{p}<0.01)$ higher levels for six VOCs (ethanol, VOC 6, acetonitrile, VOC 10, 2-butanone, VOC 18) compared to ex- and non-smokers. Acetonitrile and 2butanone are known as smoking related compounds [26]. The data for acetonitrile and 2-butanone are displayed in Figure 5a and 5b. The six compounds also correlate well with the level of exhaled carbon monoxide considering all groups as well as smokers only.

Being aware that active smoking has a pronounced effect on the VOC composition of exhaled breath, we analyzed the data separately for smokers and non/ex-smokers with respect to COPD markers. We found three VOCs that significantly differed between healthy subjects and COPD patients in the group of non/ex-smokers ( $\mathrm{p}<0.01, \mathrm{n}=9$ vs.12). These three VOCs could be also found in the group of smokers to be significantly different $(\mathrm{p}<0.01, \mathrm{n}=16 \mathrm{vs}$. 21$)$ additionally to four further VOCs. Thus, three of the 45 VOCs that were included into the analysis show a significant difference between healthy controls and patients with COPD both in the group of smokers and in the group of non/exsmokers. Two of these three VOCs rank among the 20 most abundant peaks. The two VOCs are given in Table 3 as "Compound 19 and 20" and are displayed in Figure 5c and 5d. However, the two compounds are not identified, yet. The third compound has low intensity near the chosen $\mathrm{S} / \mathrm{N}$ threshold ratio of 10 and is not identified, too. 

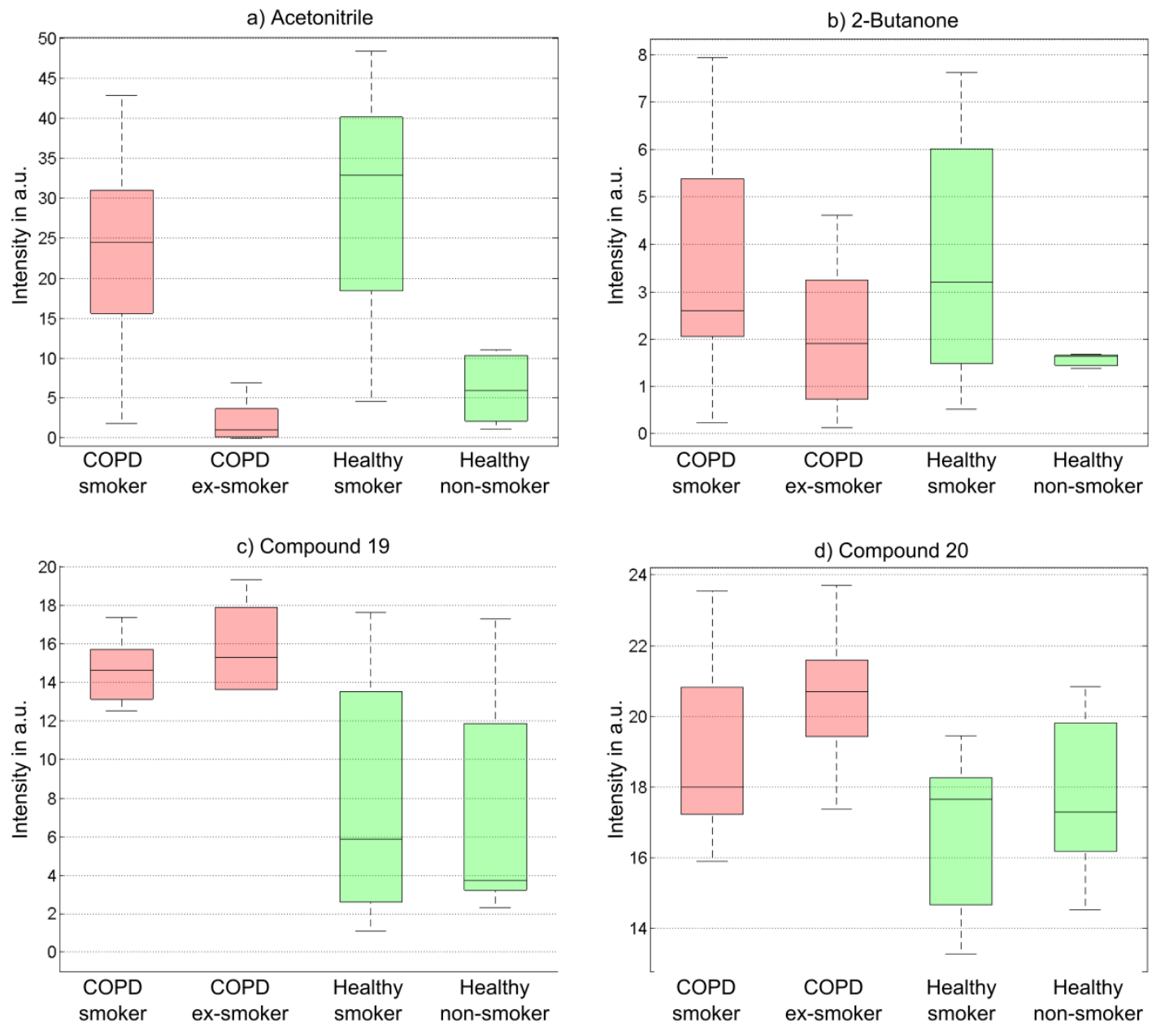

Figure 5: Box-and-whisker plots of four compounds. In a) and b) acetonitrile as well as 2-butanone is shown, clearly distinguishing active smoker from ex- and non-smoker. In c) and d) two compounds are shown significantly discriminating between COPD patients and healthy controls.

\subsection{GC-APCI-MS}

Among the 102 VOC peaks detected by GC-APCI-MS and included into the analysis, 16 were significantly different $(\mathrm{p}<0.01)$ between smokers and non/ex-smokers. Four of these compounds correlate with the level of exhaled carbon monoxide, considering all groups as well as smokers only. The two identified smoker related peaks in the GC-IMS data, acetonitrile and 2-butanone, also distinguish smokers from non/ex-smokers in the GC-APCI-MS data $(p=0.01$ and $p=0.05)$. Since other VOCs are not identified yet, they cannot be compared to the GC-IMS data.

Surprisingly, we only found one VOC that significantly differs $(\mathrm{p}<0.01)$ between healthy subjects and COPD patients in the group of non/ex-smokers ( $p=0.003, n=23$ vs.25) and in the whole group of subjects ( $\mathrm{p}=0.005, \mathrm{n}=48$ vs. 46 ). This compound was identified as 2-pentanone. In the group of smokers ( $\mathrm{n}=25$ vs. 21$)$, no VOC was found to be significantly $(\mathrm{p}<0.01)$ different in the groups.

\subsection{Comparison between GC-IMS and GC-APCI-MS data}

Investigating the correlation between GC-APCI-MS and GC-IMS data, it becomes obvious, that among the VOCs identified by reference compounds the number of close correlations is limited. For smoking related compounds, e.g. acetonitrile, the correlation is high $(r=0.73, p=1 E-8)$, but for e.g. ethanol or 2-pentanone it was found to be low $(r<0.5)$. 
For 2-pentanone the difference between controls and COPD patients was only observed in the GCAPCI-MS data. Since 2-pentanone is one of the investigated reference compounds, the GC-IMS retention time as well as the IMS drift time from 2-pentanone is known. The corresponding peak in the GC-IMS data does not significantly distinguish COPD patients from healthy controls. As 2-pentanone is the only COPD related compound found in the GC-APCI-MS data, the two compounds significantly discriminating between COPD patients and healthy controls in the GC-IMS data could not be identified as significant from the GC-APCI-MS data.

\section{Discussion}

This proof-of-principle study shows the potential of our closed gas loop, high resolution GC-IMS in clinical studies. The smoking related differences and correlations indicate that the GC-IMS data are valid. Thus, the GC-IMS provides potentially valuable diagnostic information based on VOC biomarker in exhaled breath for non-invasive diagnostics. In this study, three VOCs have been found with GC-IMS that significantly differentiate between healthy subjects and COPD patients.

The results show that the improved analytical performance of our GC-IMS is sufficient to analyze complex breath samples. The chosen standard GC-column is especially suitable for the separation of volatile organic compounds. In combination with the high-resolution ion mobility spectrometer high separation power is achieved. Furthermore, the system is very sensitive. Detection limits in the singledigit $\mathrm{ppb}_{\mathrm{v}}$ range can be reached. Almost 50 clearly separated peaks were detected in a GC-IMS breath spectrum using ultra-low sample volume of just $0.2 \mathrm{~mL}$. Thus, our GC-IMS provides sufficient analytical power for direct analysis of breath samples without any pre-concentration. The advantage of a direct analysis is that the measurement is not affected by any sample collection procedure, e.g. "selective" breath sampling on Tenax TA. Moreover, considering the instrumentation effort and relatively low cost, GC-IMS has large potential for real-time and bed-site analysis.

However, while this feasibility study clearly demonstrates the utility of GC-IMS for exhaled breath VOC analysis to discriminate COPD patients from healthy controls, some limitations of the current experimental design and analysis were recognized. For real-time online analysis, it would be desirable to reduce the current measurement time to less than 20 minutes. This time has been chosen to ensure that most breath VOCs have eluted from the GC column. Considering the GC-IMS breath measurements in this study, it is noteworthy that the majority of the VOCs have eluted after a few minutes. Hence, when using the GC-IMS in further studies, the GC parameters should be optimized with respect to separation power and measuring time. Therefore, it is possible to use a flow scheme or to vary the GC temperature. In order to better assess the possible effects, further observations are necessary.

Another focus of our work is to investigate possible correlations between low concentrated breath VOCs and COPD by using a GC-APCI-MS. Compared to our compact GC-IMS, breath measurements analyzed with GC-APCI-MS contained significantly more peaks due to the 250 -fold higher sample volume and the lower detection limits. Thus, by using the GC-APCI-MS mainly low concentrated breath VOCs are analyzed. Comparable to the GC-IMS data, several smoking related peaks have been found in the GC-APCI-MS data, but only a small fraction of these peaks correlate with the CO level. This result could be attributed to two issues. First, the linear dynamic response range of the GC-APCIMS system just reaches up to $5 \mathrm{ppb}_{\mathrm{v}}$. Since the majority of smoking related compounds in breath have higher concentrations, the GC-APCI-MS operates close to the saturation level. Thus, for higher concentrations the detector intensity becomes independent of the concentration, e.g. doubling the compound concentration does not lead to higher peak intensity. However, doubling the compound concentration possibly leads to doubling the measured $\mathrm{CO}$ level. Thus, no correlation can be detected. 
Second, due to the ultra-high sensitivity of the GC-APCI-MS system, it is possible that we detected VOCs which are no direct combustion products of smoking but instead VOCs which occur due to metabolic changes caused by smoking.

Furthermore, in the GC-APCI-MS data, we only found one VOC (2-pentanone) significantly discriminating COPD patients from healthy controls. This compound has not been found by GC-IMS. In contrast, with the GC-IMS three other VOCs are found differentiating COPD patients from healthy controls. Overall, there is just a limited number of VOCs showing close correlation between GC-IMS and GC-APCI-MS. Being aware that both measurement systems differ with respect to the linear dynamic response range, these results are not surprising. In breath, the concentration of compounds which can be detected by GC-IMS need to be significantly higher as for GC-APCI-MS. We assume that the GC-APCI-MS is in saturation for these compounds, especially when considering the preconcentration effect. Thus, no COPD discrimination can be seen in the GC-APCI-MS data for the compounds found with GC-IMS. On the other hand, the concentration of 2-pentanone in breath is comparatively low, which leads to a low signal intensity in the GC-IMS close to the noise level. Hence, it is hard to detect differences for those substances by the GC-IMS.

Apart from this, there are other reasons which also might influence the comparability of GC-IMS and GC-APCI-MS data. For example, different sample collection methods were used. For GC-IMS measurements, exhaled breath was directly analyzed. For GC-APCI-MS measurements, just endexpiratory breath was sampled on Tenax TA. Tenax TA is known to be a "selective" medium for high volatile compounds, plus, there are decomposition products interfering the discrimination from endogenous breath compounds [27]. Furthermore, alveolar air shows higher concentrations of systemic and lower concentration of exogenous substances [28]. However, this should have only a minor influence on the results.

In summary, among the low concentrated breath $\operatorname{VOCs}\left(0.1 \mathrm{ppt}_{\mathrm{v}}\right.$ to $\left.1 \mathrm{ppb}_{\mathrm{v}}\right)$, which can be measured by the GC-APCI-MS, only 2-pentanone significantly discriminates COPD patients from healthy controls. Thus, in this study, the investigation of low concentrated breath VOCs has not provided any new biomarkers discriminating COPD from healthy subjects. Most COPD related VOCs seem to appear in higher concentration ranges.

\section{Conclusion}

In this study, a novel, compact, closed gas loop, high-resolution GC-IMS and a modified ultrasensitive GC-APCI-MS were used to find COPD related biomarkers. The smoking related differences and correlations indicate that the GC-IMS data are valid. In this study, three VOCs differentiating significantly between healthy subjects and COPD patients have been found using GC-IMS. The identification of three COPD related GC-IMS signals support the use of the GC-IMS in larger COPD trials. In order to investigate possible correlations between ultra-low concentrated breath VOCs $\left(0.1 \mathrm{ppt}_{\mathrm{v}}\right.$ to $\left.1 \mathrm{ppb}_{\mathrm{v}}\right)$ and COPD, a GC-APCI-MS system has been used. Due to different linear dynamic response ranges, the data of GC-IMS and GC-APCI-MS are only comparable to a limited extent. Using GC-APCI-MS, 2-pentanone has been found to differentiate between healthy subjects and COPD patients. 


\section{References}

[1] N. Fens, van der Schee, M P, P. Brinkman, and P. J. Sterk, "Exhaled breath analysis by electronic nose in airways disease. Established issues and key questions", Clinical and experimental allergy : journal of the British Society for Allergy and Clinical Immunology, vol. 43, no. 7, pp. 705-715, 2013.

[2] Y.-J. Lin, H.-R. Guo, Y.-H. Chang, M.-T. Kao, H.-H. Wang, and R.-I. Hong, "Application of the electronic nose for uremia diagnosis", Sensors and Actuators B: Chemical, vol. 76, no. 1-3, pp. 177-180, 2001.

[3] T. Ligor, M. Ligor, A. Amann, C. Ager, M. Bachler, A. Dzien, and B. Buszewski, "The analysis of healthy volunteers' exhaled breath by the use of solid-phase microextraction and GC-MS", Journal of breath research, vol. 2, no. 4, p. 046006, 2008.

[4] W. Miekisch, P. Fuchs, S. Kamysek, C. Neumann, and J. K. Schubert, "Assessment of propofol concentrations in human breath and blood by means of HS-SPME-GC-MS", Clinica chimica acta; international journal of clinical chemistry, vol. 395, no. 1-2, pp. 32-37, 2008.

[5] P. Spaněl and D. Smith, "Progress in SIFT-MS: breath analysis and other applications", Mass Spectrom Rev, vol. 30, no. 2, pp. 236-267, 2011.

[6] W. Lindinger, A. Hansel and A. Jordan, "On-line monitoring of volatile organic compounds at pptv levels by means of proton-transfer-reaction mass spectrometry (PTR-MS) - Medical applications, food control and environmental research.", Int J Mass Spectrom 173: 191-241, 1998.

[7] B. Moser, F. Bodrogi, G. Eibl, M. Lechner, J. Rieder, and P. Lirk, "Mass spectrometric profile of exhaled breath--field study by PTR-MS", Respiratory physiology \& neurobiology, vol. 145, no. 23, pp. 295-300, 2005.

[8] J. I. Baumbach, "Ion mobility spectrometry coupled with multi-capillary columns for metabolic profiling of human breath", Journal of breath research, vol. 3, no. 3, p. 034001, 2009.

[9] A. Wolf, J. I. Baumbach, A. Kleber, F. Maurer, S. Maddula, P. Favrod, M. Jang, T. Fink, T. Volk, and S. Kreuer, "Multi-capillary column-ion mobility spectrometer (MCC-IMS) breath analysis in ventilated rats: a model with the feasibility of long-term measurements", Journal of breath research, vol. 8, no. 1, p. 016006, 2014.

[10] Van Berkel, J J B N, J. W. Dallinga, G. M. Möller, Godschalk, R W L, E. J. Moonen, Wouters, E F $\mathrm{M}$, and Van Schooten, F J, "A profile of volatile organic compounds in breath discriminates COPD patients from controls", Respiratory medicine, vol. 104, no. 4, pp. 557-563, 2010.

[11] C. Phillips, N. Mac Parthaláin, Y. Syed, D. Deganello, T. Claypole, and K. Lewis, "Short-Term Intra-Subject Variation in Exhaled Volatile Organic Compounds (VOCs) in COPD Patients and Healthy Controls and Its Effect on Disease Classification", Metabolites, vol. 4, no. 2, pp. 300318, 2014.

[12] N. Fens, A. H. Zwinderman, van der Schee, Marc P, de Nijs, Selma B, E. Dijkers, A. C. Roldaan, D. Cheung, E. H. Bel, and P. J. Sterk, "Exhaled Breath Profiling Enables Discrimination of Chronic Obstructive Pulmonary Disease and Asthma," Am J Respir Crit Care Med, vol. 180, no. 11, pp. 1076-1082, 2009.

[13] S. Dragonieri, J. T. Annema, R. Schot, van der Schee, Marc P C, A. Spanevello, P. Carratú, O. Resta, K. F. Rabe, and P. J. Sterk, "An electronic nose in the discrimination of patients with nonsmall cell lung cancer and COPD", Lung cancer (Amsterdam, Netherlands), vol. 64, no. 2, pp. 166-170, 2009.

[14] M. Westhoff, P. Litterst, S. Maddula, B. Bödeker, S. Rahmann, A. N. Davies, and J. I. Baumbach, "Differentiation of chronic obstructive pulmonary disease (COPD) including lung cancer from 
healthy control group by breath analysis using ion mobility spectrometry," Int. J. Ion Mobil. Spec, vol. 13, no. 3-4, pp. 131-139, 2010.

[15] P. Zarogoulidis, L. Freitag, V. Besa, H. Teschler, I. Kurth, A. M. Khan, U. Sommerwerck, I. Baumbach, and K. Darwiche, "Exhaled volatile organic compounds discriminate patients with chronic obstructive pulmonary disease from healthy subjects," COPD, p. 399, 2015.

[16] V. Ruzsanyi, J. I. Baumbach, S. Sielemann, P. Litterst, M. Westhoff, and L. Freitag, "Detection of human metabolites using multi-capillary columns coupled to ion mobility spectrometers," Journal of Chromatography A, vol. 1084, no. 1-2, pp. 145-151, 2005.

[17] C. B. Hariharan, Implementation of multi capillary column ion mobility spectrometry (MCC-IMS) for medical and biological applications. Dissertation, 2012.

[18] J. Langejuergen, C. Wagner, S. Beutel, T. Hopmeier, T. Scheper, and S. Zimmermann, “Noninvasive monitoring of bacterial growth and auto-induced protein production in a bioreactor with a closed-loop GC-IMS," Int. J. Ion Mobil. Spec, vol. 18, no. 1-2, pp. 9-15, 2015.

[19] A. T. Kirk, M. Allers, P. Cochems, J. Langejuergen, and S. Zimmermann, "A compact high resolution ion mobility spectrometer for fast trace gas analysis", The Analyst, vol. 138, no. 18, pp. 5200-5207, 2013.

[20] J. Langejuergen, A. Gaida, O. Holz, S. Schuchardt, J.-M. Hohlfeld, and S. Zimmermann, Ultrasensitive atmospheric pressure chemical ionization mass spectrometry for breath analysis, International Association of Breath Research IABR - Summit '14, p. 116, Torun, Poland, July 2014.

[21] J. Langejuergen, T. Hopmeier, S. Meinen, E. Carstens, G. Theilmeier, and W. Koppert, S. Zimmermann, Analysis of exhaled breath during surgery, Int. Meeting on Chemical Sensors IMCS '12, pp. 588-591, Nuremberg, Germany, May 2012.

[22] C. J. Proctor and Todd, John F. J, "Atmospheric pressure ionization mass spectrometry," Org. Mass Spectrom, vol. 18, no. 12, pp. 509-516, 1983.

[23] A. Gaida, O. Holz, C. Nell, S. Schuchardt, B. Lavae-Mokhtari, L. Kruse, U. Boas, Langejuergen J, M. Allers, S. Zimmermann, C. Vogelmeier, A.-R. Koczulla, and J.-M. Hohlfeld, "A dual center study to compare breath volatile organic compounds from smokers and non-smokers with and without COPD", Journal of breath research, 2016.

[24] J. Langejürgen, Entwicklung und Realisierung kompakter Messsysteme zur quantitativen Detektion von organischen Spurengasen in Luft, 1st ed. Herzogenrath: Shaker, 2015.

[25] J. Langejuergen, P. Cochems, and S. Zimmermann, Online trace gas detection - pushing a Bruker micrOTOF II towards ppq-detection limits using simple atmospheric pressure ionization sources, Messtechnisches Symposium des Arbeitskreises der Hochschullehrer für Messtechnik - AHMT , pp. 199-210, Karlsruhe, Germany, September 2011.

[26] B. Buszewski, A. Ulanowska, T. Ligor, N. Denderz, and A. Amann, "Analysis of exhaled breath from smokers, passive smokers and non-smokers by solid-phase microextraction gas chromatography/mass spectrometry", Biomedical chromatography : BMC, vol. 23, no. 5, pp. 551-556, 2009.

[27] J. H. Lee, S. A. Batterman, C. Jia, and S. Chernyak, "Ozone artifacts and carbonyl measurements using Tenax GR, Tenax TA, Carbopack B, and Carbopack X adsorbents", Journal of the Air \& Waste Management Association (1995), vol. 56, no. 11, pp. 1503-1517, 2006.

[28] W. Miekisch, S. Kischkel, A. Sawacki, T. Liebau, M. Mieth, and J. K. Schubert, "Impact of sampling procedures on the results of breath analysis", Journal of breath research, vol. 2, no. 2, p. $026007,2008$. 